\title{
Analytical solutions of the equations for the transient temperature field in the three-fluid parallel-channel heat exchanger with three thermal communications
}

\author{
Leszek Malinowski • Jian-Hua Chen
}

Received: date / Accepted: date

\begin{abstract}
The partial differential equations for the general case of the threefluid parallel-channel heat exchanger are solved analytically by formulating them into boundary control systems. Both the co- and the counter-flow cases with three thermal communications between the channels are considered. Based on the analytical solution formula for the transient case, the steady state and the time to reach the steady state are studied. Illustrating examples and numerical simulations are given.
\end{abstract}

Keywords three-fluid parallel-channel heat exchanger $\cdot$ co-flow $\cdot$ counterflow $\cdot$ analytical solution $\cdot$ transient case

\section{Introduction}

As important components of numerous thermal systems, heat exchangers are widely used in a variety of industrial processes and engineering experiments to realize the transfer of heat between hot and cold fluids or gases [11], for instance in: refrigeration, air conditioning, power plants, chemical plants, petrochemical plants, petroleum refineries, natural gas processing, and sewage treatment. Among which, the three-fluid parallel-flow and the three-fluid counter-flow heat exchangers or the mixed ones, are popular and commonly utilized in many

Leszek Malinowski

Faculty of Maritime Technology and Transport

West Pomeranian University of Technology

Al. Piastów 41, Szczecin 71-065, Poland

E-mail: leszek.malinowski@zut.edu.pl

Jian-Hua Chen

School of Mathematics and Computational Science

Hunan University of Science and Technology

Xiangtan, Hunan 411201, PR China

E-mail: cjhmath@mail.ustc.edu.cn 
places, such as: helium-air separation units, ammonia gas synthesis, hydrogen liquefaction processes, and purification systems.

In this paper, we are concerned with the following equations for the threefluid parallel-channel heat exchanger with three thermal communications

$$
\left\{\begin{array}{l}
\frac{\partial T_{1}}{\partial t}(x, t)+s_{1} v_{1} \frac{\partial T_{1}}{\partial x}(x, t)=b_{12}\left[T_{2}(x, t)-T_{1}(x, t)\right]+b_{13}\left[T_{3}(x, t)-T_{1}(x, t)\right], \\
\frac{\partial T_{2}}{\partial t}(x, t)+s_{2} v_{2} \frac{\partial T_{2}}{\partial x}(x, t)=b_{21}\left[T_{1}(x, t)-T_{2}(x, t)\right]+b_{23}\left[T_{3}(x, t)-T_{2}(x, t)\right], \\
\frac{\partial T_{3}}{\partial t}(x, t)+s_{3} v_{3} \frac{\partial T_{3}}{\partial x}(x, t)=b_{31}\left[T_{1}(x, t)-T_{3}(x, t)\right]+b_{32}\left[T_{2}(x, t)-T_{3}(x, t)\right],
\end{array}\right.
$$

Here, $T_{i}(x, t)$ denotes the temperature of the fluid in the channel $i$ at time $t$ and at position $0 \leq x \leq l$, the letter $l$ represents the length of the heat exchanger, $s_{i}= \pm 1$ indicates the direction of the fluid $i$, the positive constants $b_{i j}$ are of dimension $\mathrm{Hz}$,

$$
b_{i j}=\frac{k_{i j} P_{i j}}{\rho_{i} c_{p i} A_{i}}
$$

where $k_{i j}=k_{j i}, P_{i j}=P_{j i}(i, j=1,2,3 ; i \neq j)$. Note that strictly speaking, each $b_{i j}$ depends on other physical coefficients that depend on temperature and hence on position and time. The constant assumption is an approximation which makes the problem easy to deal with.

\section{Nomenclature.}

\begin{tabular}{ll}
\hline symbol & physical meaning and dimension \\
\hline$A_{i}$ & cross section area of channel $i, \mathrm{~m}^{2}$ \\
$b_{i j}$ & quantities given by $(2), \mathrm{Hz}$ \\
$c_{p i}$ & specific heat of fluid $i$ at constant pressure, $\mathrm{J} /(\mathrm{kg} \cdot \mathrm{K})$ \\
$H^{1}(0, l)$ & the first order Sobolev function space \\
$k_{i j}$ & overall heat transfer coefficient between channels \\
& $i$ and $j, \mathrm{~W} / \mathrm{m} \cdot \mathrm{K})$ \\
$l$ & common length of the channels, $\mathrm{m}$ \\
$L^{2}(0, l)$ & the function space of space of squarely integrable functions \\
$P_{i j}$ & common perimeter of channels $i$ and $j, \quad \mathrm{~m}$ \\
$s_{i}$ & flow direction of fluid $i, s_{i}=1$ means positive $x$-direction and \\
$S_{i}(t)$ & $s_{i}=-1$ means negative $x$-direction \\
$\mathbb{S}_{l}(t)$ & the $i$-th term of the Dyson-Philips series \\
$\mathbb{S}_{r}(t)$ & the left-shift semigroup on $L^{2}(0, l)$ \\
$t$ & time,,$\quad \mathrm{s}$ \\
$T_{i}(x, t)$ & the transient temperature profiles, ${ }^{\circ} \mathrm{C}$ \\
$\mathbb{T}(t)$ & the system operator semigroup \\
$u_{i}(t)$ & the system boundary input into channel $i$ \\
$v_{i}$ & velocity of the fluid in channel $i$, \\
$x$ & Cartesian coordinate, $\mathrm{m} / \mathrm{s}$ \\
$Z$ & $Z=L^{2}(0, l) \times L^{2}(0, l) \times L^{2}(0, l)$, a product space \\
$\phi_{i}(x)$ & initial temperature fields of the fluid $i$ \\
$\rho_{i}$ & density of the fluid $i, \quad \mathrm{~kg} / \mathrm{m}{ }^{3}$
\end{tabular}


The above type of heat exchanger equations has been studied in $[1$, Section 2 ] where it was assumed that the heat transfer coefficients and the physical properties of the fluids as well as the walls are constant. The fluid temperature and mass flow rate in each channel were supposed to be uniform on the cross section perpendicular to the stream direction. In addition, unlike [10], the axial heat diffusion in the walls and the heat capacity of the walls were not taken into account.

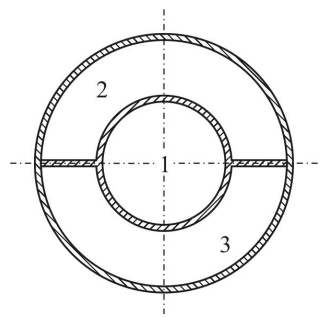

Fig. 1 Cross section of the heat exchanger with three thermal connections.

The transient temperature fields in multi-channel heat exchangers and related topics have received much attention in recent years [1, 2, 5, 8-10, 13, 14]. Different methods have been adopted and different topics have been concerned. Explicit solutions of the equations for the three-fluid heat exchanger at steady states have been derived for all fluid flow arrangements [15], see Figure 1 therein for all the possible arrangements. Under some additional conditions, an analytical solution was obtained in [1] applying the Laplace transform method. However, to the knowledge of the authors, what obtained in the literature are mostly either numerical solutions or the analytical stationary field solution. In order to obtain stationary initial conditions and stationary final state for (1), its stationary case is solved with relevant boundary conditions. Well suitable for this purpose is the effective shooting method [6].

Recently, we solved analytically the three-fluid parallel-flow heat exchanger equation with two thermal communications for the case $s_{1}=s_{2}=s_{3}=1$ by formulating it into a boundary control system, see [2] for the details. In the present paper, this method is adopted again to study the general case of equations for the three-fluid parallel-channel heat exchanger with three thermal communications.

The rest of this paper is organized as follows. In Section 2, we solve (1) analytically. Based on the analytical solutions, the steady state and the steady state time are then derived which follows from the nilpotence of the underlying system semigroup. In Section 3, illustrating examples and numerical simulations are given. Section 4 is devoted to conclusions. 


\section{Analytical solutions}

In this section, we solve (1) analytically. As mentioned before, the state space approach is adopted and we work in the real state space

$$
Z:=L^{2}(0, l) \times L^{2}(0, l) \times L^{2}(0, l)
$$

where $L^{2}(0, l)$ stands for the space of squarely integrable functions on $(0, l)$. The standing assumptions on the initial temperature fields $T_{i}(x, 0)=\phi_{i}(x)$ and the boundary inputs $u_{i}(t)$ are

$$
\phi_{i} \in H^{1}(0, l), \quad u_{i}(\cdot) \in C^{2}[0, \infty), \quad i=1,2,3
$$

where $H^{1}(0, l)$ is the first order Sobolev function space (differentiable functions in the sense of distribution, equivalently, absolutely continuous and hence differentiable almost everywhere with derivative in $L^{2}(0, l)$; we refer to any a standard textbook on functional analysis, for instance [12, Section 7.2] for more information).

All the following possible fluid flow arrangements will be considered (note that Case 1 with two thermal communication has been studied in [2]).

Case 1: $s_{1}=s_{2}=s_{3}=1$.

Case 2: $s_{1}=s_{3}=1, \quad s_{2}=-1$.

Case 3: $s_{1}=1, \quad s_{2}=s_{3}=-1$.

Case 4: $s_{1}=s_{2}=1, \quad s_{3}=-1$.

The boundary conditions are possible combinations of $T_{i}(0, t)=u_{i}(t)$ and $T_{j}(l, t)=u_{j}(t)$. The concrete form depends on the fluid flow arrangement. For example, the boundary conditions corresponding to Case 2 are

$$
T_{1}(0, t)=u_{1}(t), \quad T_{2}(l, t)=u_{2}(t), \quad T_{3}(0, t)=u_{3}(t) .
$$

As a state space method, the boundary control system method is classical and effective in solving linear partial differential equations with boundary inputs. In the following, we solve (1) and (3) case by case. Since the discussions are in parallel to those in [2], here we only state the results. Let $\mathbb{S}_{r}(t)$ and $\mathbb{S}_{l}(t)$ be the right-shift and left-shift semigroups on $L^{2}(0, l)$ given by

$$
\begin{gathered}
\left(\mathbb{S}_{r}(t) f\right)(x)=\left\{\begin{array}{l}
f(x-t), \quad x \geq t \\
0, \quad \text { otherwise }
\end{array}\right. \\
\left(\mathbb{S}_{l}(t) f\right)(x)= \begin{cases}f(x+t), & x+t \leq l, \\
0, & \text { otherwise }\end{cases}
\end{gathered}
$$


2.1 Analytical solution for Case 1

For Case 1, the boundary conditions are

$$
T_{1}(0, t)=u_{1}(t), \quad T_{2}(0, t)=u_{2}(t), \quad T_{3}(0, t)=u_{3}(t)
$$

if

$$
\phi_{1}(0)=u_{1}(0), \quad \phi_{2}(0)=u_{2}(0), \quad \phi_{3}(0)=u_{3}(0),
$$

then (1) with $s_{1}=s_{2}=s_{3}=1$ subject to (3) and (7) has the solution

$$
\begin{aligned}
{\left[\begin{array}{l}
T_{1}(x, t) \\
T_{2}(x, t) \\
T_{3}(x, t)
\end{array}\right] } & =\left[\begin{array}{l}
(x+1) u_{1}(t) \\
(x+1) u_{2}(t) \\
(x+1) u_{3}(t)
\end{array}\right]+\mathbb{T}(t)\left[\begin{array}{l}
\phi_{1}(x)-(x+1) u_{1}(0) \\
\phi_{2}(x)-(x+1) u_{2}(0) \\
\phi_{3}(x)-(x+1) u_{3}(0)
\end{array}\right] \\
+ & \int_{0}^{t} \mathbb{T}(t-s)\left[\begin{array}{l}
U_{11}(x, s) \\
U_{12}(x, s) \\
U_{13}(x, s)
\end{array}\right] \mathrm{d} s .
\end{aligned}
$$

Here,

$$
\begin{aligned}
U_{11}(x, s)= & -\left[v_{1}+\left(b_{12}+b_{13}\right)(x+1)\right] u_{1}(s)-(x+1) \dot{u}_{1}(s) \\
& +b_{12}(x+1) u_{2}(s)+b_{13}(x+1) u_{3}(s), \\
U_{12}(x, s)= & -\left[v_{2}+\left(b_{21}+b_{23}\right)(x+1)\right] u_{2}(s)-(x+1) \dot{u}_{2}(s) \\
& +b_{21}(x+1) u_{1}(s)+b_{23}(x+1) u_{3}(s), \\
U_{13}(x, s)= & -\left[v_{3}+\left(b_{31}+b_{32}\right)(x+1)\right] u_{3}(s)-(x+1) \dot{u}_{3}(s) \\
& +b_{31}(x+1) u_{1}(s)+b_{32}(x+1) u_{2}(s),
\end{aligned}
$$

$\mathbb{T}(t)$ represents the strongly continuous operator semigroup (simply, operator semigroup, this is a concept in functional analysis and an important tool to solve linear partial differential equations, see the monograph [12] for more information) on $Z$ which is given by the following Dyson-Phillips series

$$
\begin{aligned}
& \mathbb{T}(t)=\sum_{n=0}^{\infty} S_{n}(t) \\
& P:=\left[\begin{array}{ccc}
0 & b_{12} & b_{13} \\
b_{21} & 0 & b_{23} \\
b_{31} & b_{32} & 0
\end{array}\right], \quad S_{n+1}(t) f=\int_{0}^{t} S_{n}(t-s) P S_{0}(s) f \mathrm{~d} s, \quad f \in Z, \\
& S_{0}(t)=\left[\begin{array}{ccc}
\mathrm{e}^{-\left(b_{12}+b_{13}\right) t} \mathbb{S}_{r}\left(v_{1} t\right) & 0 & 0 \\
0 & \mathrm{e}^{-\left(b_{21}+b_{23}\right) t} \mathbb{S}_{r}\left(v_{2} t\right) & 0 \\
0 & 0 & \mathrm{e}^{-\left(b_{31}+b_{32}\right) t} \mathbb{S}_{r}\left(v_{3} t\right)
\end{array}\right] \text {. }
\end{aligned}
$$


2.2 Analytical solution for Case 2

For Case 2, the boundary conditions are (4), if

$$
\phi_{1}(0)=u_{1}(0), \quad \phi_{2}(l)=u_{2}(0), \quad \phi_{3}(0)=u_{3}(0),
$$

then (1) with $s_{1}=s_{3}=1$ and $s_{2}=-1$ subject to (3) and (4) has the solution

$$
\begin{aligned}
{\left[\begin{array}{l}
T_{1}(x, t) \\
T_{2}(x, t) \\
T_{3}(x, t)
\end{array}\right] } & =\left[\begin{array}{c}
(x+1) u_{1}(t) \\
\frac{x}{l} u_{2}(t) \\
(x+1) u_{3}(t)
\end{array}\right]+\mathbb{T}(t)\left[\begin{array}{c}
\phi_{1}(x)-(1+x) u_{1}(0) \\
\phi_{2}(x)-\frac{x}{l} u_{2}(l) \\
\phi_{3}(x)-(1+x) u_{3}(0)
\end{array}\right] \\
& +\int_{0}^{t} \mathbb{T}(t-s)\left[\begin{array}{c}
U_{21}(x, s) \\
U_{22}(x, s) \\
U_{23}(x, s)
\end{array}\right] \mathrm{d} s .
\end{aligned}
$$

Here,

$$
\begin{gathered}
U_{21}(x, s)=-\left[v_{1}+\left(b_{12}+b_{13}\right)(x+1)\right] u_{1}(s)-(x+1) \dot{u}_{1}(s) \\
+\frac{b_{12} x}{l} u_{2}(s)+b_{13}(x+1) u_{3}(s), \\
U_{22}(x, s)=\frac{v_{2}-\left(b_{21}+b_{23}\right) x}{l} u_{2}(s)-\frac{x}{l} \dot{u}_{2}(s) \\
+b_{21}(x+1) u_{1}(s)+b_{23}(x+1) u_{3}(s), \\
U_{23}(x, s)=-\left[v_{3}+\left(b_{31}+b_{32}\right)(x+1)\right] u_{3}(s)-(x+1) \dot{u}_{3}(s) \\
+b_{31}(x+1) u_{1}(s)+\frac{b_{32} x}{l} u_{2}(s),
\end{gathered}
$$

$\mathbb{T}(t)$ is given by (10) with

$$
S_{0}(t)=\left[\begin{array}{ccc}
\mathrm{e}^{-\left(b_{12}+b_{13}\right) t} \mathbb{S}_{r}\left(v_{1} t\right) & 0 & 0 \\
0 & \mathrm{e}^{-\left(b_{21}+b_{23}\right) t} \mathbb{S}_{l}\left(v_{2} t\right) & 0 \\
0 & 0 & \mathrm{e}^{-\left(b_{31}+b_{32}\right) t} \mathbb{S}_{r}\left(v_{3} t\right)
\end{array}\right] .
$$

2.3 Analytical solution for Case 3

For Case 3, the boundary conditions are

$$
T_{1}(0, t)=u_{1}(t), \quad T_{2}(l, t)=u_{2}(t), \quad T_{3}(l, t)=u_{3}(t),
$$

if

$$
\phi_{1}(0)=u_{1}(0), \quad \phi_{2}(l)=u_{2}(0), \quad \phi_{3}(l)=u_{3}(0),
$$


the solution of (1) with $s_{1}=1$ and $s_{2}=s_{3}=-1$ subject to (3) and (13) is

$$
\begin{aligned}
{\left[\begin{array}{l}
T_{1}(x, t) \\
T_{2}(x, t) \\
T_{3}(x, t)
\end{array}\right] } & =\left[\begin{array}{c}
(x+1) u_{1}(t) \\
\frac{x}{l} u_{2}(t) \\
\frac{x}{l} u_{3}(t)
\end{array}\right]+\mathbb{T}(t)\left[\begin{array}{c}
\phi_{1}-(1+x) u_{1}(0) \\
\phi_{2}-\frac{x}{l} u_{2}(l) \\
\phi_{3}-\frac{x}{l} u_{3}(l)
\end{array}\right] \\
& +\int_{0}^{t} \mathbb{T}(t-s)\left[\begin{array}{c}
U_{31}(x, s) \\
U_{32}(x, s) \\
U_{33}(x, s)
\end{array}\right] \mathrm{d} s .
\end{aligned}
$$

Here,

$$
\begin{gathered}
U_{31}(x, s)=-\left[v_{1}+\left(b_{12}+b_{13}\right)(x+1)\right] u_{1}(s)-(x+1) \dot{u}_{1}(s) \\
+\frac{x}{l}\left[b_{12} u_{2}(s)+b_{13} u_{3}(s)\right], \\
U_{32}(x, s)=\frac{v_{2}-\left(b_{21}+b_{23}\right) x}{l} u_{2}(s)-\frac{x}{l} \dot{u}_{2}(s) \\
+b_{21}(x+1) u_{1}(s)+\frac{b_{23} x}{l} u_{3}(s), \\
U_{33}(x, s)= \\
\quad \frac{v_{3}-\left(b_{31}+b_{32}\right) x}{l} u_{3}(s)-\frac{x}{l} \dot{u}_{3}(s) \\
+b_{31}(x+1) u_{1}(s)+\frac{b_{32} x}{l} u_{2}(s),
\end{gathered}
$$

$\mathbb{T}(t)$ is given by (10) with

$$
S_{0}(t)=\left[\begin{array}{ccc}
\mathrm{e}^{-\left(b_{12}+b_{13}\right) t} \mathbb{S}_{r}\left(v_{1} t\right) & 0 & 0 \\
0 & \mathrm{e}^{-\left(b_{21}+b_{23}\right) t} \mathbb{S}_{l}\left(v_{2} t\right) & 0 \\
0 & 0 & \mathrm{e}^{-\left(b_{31}+b_{32}\right) t} \mathbb{S}_{l}\left(v_{3} t\right)
\end{array}\right] .
$$

\subsection{Analytical solution for Case 4}

For Case 4, the boundary conditions are

$$
T_{1}(0, t)=u_{1}(t), \quad T_{2}(0, t)=u_{2}(t), \quad T_{3}(l, t)=u_{3}(t),
$$

if

$$
\phi_{1}(0)=u_{1}(0), \quad \phi_{2}(0)=u_{2}(0), \quad \phi_{3}(l)=u_{3}(0),
$$

then (1) with $s_{1}=s_{2}=1$ and $s_{3}=-1$ subject to (3) and (15) has the solution

$$
\begin{aligned}
{\left[\begin{array}{c}
T_{1}(x, t) \\
T_{2}(x, t) \\
T_{3}(x, t)
\end{array}\right] } & =\left[\begin{array}{c}
(x+1) u_{1}(t) \\
(x+1) u_{2}(t) \\
\frac{x}{l} u_{3}(t)
\end{array}\right]+\mathbb{T}(t)\left[\begin{array}{c}
\phi_{1}(x)-(x+1) u_{1}(0) \\
\phi_{2}(x)-(x+1) u_{2}(0) \\
\phi_{3}(x)-\frac{x}{l} u_{3}(l)
\end{array}\right] \\
& +\int_{0}^{t} \mathbb{T}(t-s)\left[\begin{array}{c}
U_{41}(x, s) \\
U_{42}(x, s) \\
U_{43}(x, s)
\end{array}\right] \mathrm{d} s .
\end{aligned}
$$


Here,

$$
\begin{aligned}
& U_{41}(x, s)=-\left[v_{1}+\left(b_{12}+b_{13}\right)(x+1)\right] u_{1}(s)-(x+1) \dot{u}_{1}(s) \\
& +b_{12}(x+1) u_{2}(s)+\frac{b_{13} x}{l} u_{3}(s) \text {, } \\
& U_{42}(x, s)=-\left[v_{2}+\left(b_{21}+b_{23}\right)(x+1)\right] u_{2}(s)-(x+1) \dot{u}_{2}(s) \\
& +b_{21}(x+1) u_{1}(s)+\frac{b_{23} x}{l} u_{3}(s), \\
& U_{43}(x, s)=\frac{v_{3}-\left(b_{31}+b_{32}\right) x}{l} u_{3}(s)-\frac{x}{l} \dot{u}_{3}(s)+\left[b_{31} u_{1}(s)+b_{32} u_{2}(s)\right](x+1),
\end{aligned}
$$

$\mathbb{T}(t)$ is given by (10) with

$$
S_{0}(t)=\left[\begin{array}{ccc}
\mathrm{e}^{-\left(b_{12}+b_{13}\right) t} \mathbb{S}_{r}\left(v_{1} t\right) & 0 & 0 \\
0 & \mathrm{e}^{-\left(b_{21}+b_{23}\right) t} \mathbb{S}_{r}\left(v_{2} t\right) & 0 \\
0 & 0 & \mathrm{e}^{-\left(b_{31}+b_{32}\right) t} \mathbb{S}_{l}\left(v_{3} t\right)
\end{array}\right] .
$$

2.5 Steady states and steady state time

In this subsection, as done in [2, Section 3], assuming that the input functions $u_{i}(t)$ do not change for $t$ sufficiently large (without loss of generality, we may assume that the inputs are constant), we derive the system steady states and the time for the heat exchanger to reach the steady state using the obtained analytical solution formulas.

Analogously, it can be shown that the systems will reach the steady states and from the mathematical point of view, this is because of the nilpotency of the system semigroups. Recall that an operator semigroup $\mathbb{T}(t)$ is called nilpotent if there exists a constant $r>0$ satisfying

$$
\mathbb{T}(t)=0, \quad \forall t>r
$$

and it is obvious that the right-shift and left-shift semigroups $\mathbb{S}_{r}(t)$ and $\mathbb{S}_{l}(t)$ in (5) and (6) are nilpotent, more precisely,

$$
\mathbb{S}_{r}(t)=0=\mathbb{S}_{l}(t), \quad t>l .
$$

The mathematical proof is similar to (but the derivation is a little bit more difficult) that in [2, Section 3.1] and is omitted, only the results are given here. Roughly speaking, the nilpotency of the system operator semigroups inherits from that of (17). For Cases 1-4, the common steady state time is

$$
t_{s}=\max \left\{\frac{l\left(v_{2}+v_{3}\right)}{v_{2} v_{3}}, \frac{l\left(v_{1}+v_{3}\right)}{v_{1} v_{3}}, \frac{l\left(v_{1}+v_{2}\right)}{v_{1} v_{2}}\right\} .
$$

We note that $l\left(v_{i}+v_{j}\right) /\left(v_{i} v_{j}\right)=l / v_{i}+l / v_{j}$. Therefore, as compared with the two thermal connection case (the steady state time is $l / v_{\min }$ with $v_{\min }=$ 
$\left.\min \left\{v_{1}, v_{2}, v_{3}\right\}\right)$, it is obvious that more time is needed for the systems to reach the steady states, which is intuitively reasonable. In addition, letting

$$
u_{i}(t)=\nu_{i}, \quad i=1,2,3,
$$

it is not hard to compute the steady states. For example, the steady state of Case 4 is

$$
\left[\begin{array}{c}
T_{s 1}(x) \\
T_{s 2}(x) \\
T_{s 3}(x)
\end{array}\right]=\left[\begin{array}{c}
(x+1) \nu_{1} \\
(x+1) \nu_{2} \\
\frac{x}{l} \nu_{3}
\end{array}\right]+\int_{0}^{t_{s}} \mathbb{T}(\tau)\left[\begin{array}{l}
U_{41}(x) \\
U_{42}(x) \\
U_{43}(x)
\end{array}\right] \mathrm{d} s,
$$

where $\mathbb{T}$ is as in Section 2.4 and

$$
\begin{gathered}
U_{41}(x)=-\left[v_{1}+\left(b_{12}+b_{13}\right)(x+1)\right] \nu_{1}+(x+1) b_{12} \nu_{2}+\frac{\nu_{3} b_{13} x}{l} \\
U_{42}(x)=-\left[v_{2}+\left(b_{21}+b_{23}\right)(x+1)\right] \nu_{2}+(x+1) b_{21} \nu_{1}+\frac{\nu_{3} b_{23} x}{l} \\
U_{43}(x)=\frac{v_{3}-\left(b_{31}+b_{32}\right) x}{l} \nu_{3}+(x+1)\left[b_{31} \nu_{1}+b_{32} \nu_{2}\right] .
\end{gathered}
$$

\section{Numerical simulations}

In this section, for the special case of constant inputs, we solve (1) and (2) numerically to observe the time to reach the steady state. Three examples are calculated, concerning Cases 2-4 (Case 1 is similar to [2] and hence not considered here), see their characterizations in Table 3. The formula (18) is verified by the numerical simulations which in turn validates the adopted numerical method.

The calculations are implemented using the MathCad software version 15. In order to determine the steady state temperature field the Odesolve function is applied (Adams/BDF method). The transient temperature field is calculated using the Pdesolve function (polynomial method). The thermophysical properties of water are determined for the arithmetic mean of the minimum and maximum temperature in the exchanger. Since the effect of the fluid velocity on the overall heat transfer coefficients is taken into account, two sets of $b_{i j}$ are adopted (see Table 2) and the second set is especially for Example 2 due to the change of the fluid velocity in channel 2 .

The numerical results for the three examples specified in Table 3 are presented in Figs. 2-4. Three temperature profiles are plotted for each channel. It is observed that the transient temperature profile tends to overlap the final steady state profile as the time elapses. A temperature jump inside channel 3 (Example 3) is not smoothed due to the lack of longitudinal heat transfer in the fluid in the model given by (1). The convergence rate depends on the initial and boundary conditions. It is faster for Example 2 then for Example 1. The slowest convergence rate is observed in Example 3 due to the temperature 
Table 1 Data for calculations.

\begin{tabular}{clc}
\hline parameter & dimension & value \\
\hline inner tube inside diameter & $\mathrm{m}$ & 0.06 \\
outer tube inside diameter & $\mathrm{m}$ & 0.08 \\
channels length & $\mathrm{m}$ & 2 \\
tube wall thickness & $\mathrm{m}$ & 0.0015 \\
wype of fluids in the three channels & & 50 \\
heat conductivity of walls & $W /(\mathrm{m} \cdot \mathrm{K})$ & \\
fluid velocities & $\mathrm{m} / \mathrm{s}$ & 0.5 \\
channel 1 & & 1 \\
channel 2 & & 0.6 \\
channel 3 & & \\
inlet fluid temperatures & $\mathrm{C}$ & 30 \\
channel 1 & & 70 \\
channel 2 & & 10 \\
channel 3 & &
\end{tabular}

Table 2 Two sets of $b_{i j}$. Set 2 is only for Example 2 because of the fluid velocity change in channel 2 .

\begin{tabular}{clllllc}
\hline set & $b_{12}$ & $b_{21}$ & $b_{23}$ & $b_{32}$ & $b_{13}$ & $b_{31}$ \\
\hline 1 & 0.0396546 & 0.0396546 & 0.1375363 & 0.0985952 & 0.1088912 & 0.0779172 \\
2 & 0.0358552 & 0.0358552 & 0.1230281 & 0.0881948 & 0.1088912 & 0.0779172 \\
\hline
\end{tabular}

Table 3 Characterizations of computed examples.

\begin{tabular}{cl}
\hline example & characterization \\
1 & $\begin{array}{l}\text { flow arrangement Case } 2, \text { constant inlet temperatures and constant } \\
\text { initial temperatures with } T_{i}(0, t)=T_{i}(x, 0) \equiv T_{i 0}\end{array}$ \\
& flow arrangement Case 3, constant inlet temperatures, the initial \\
& state is the steady state $\left(b_{i j}\right.$ are as in Set 1$)$, step decrease in fluid \\
& flow in channel 2 by $0.3 \mathrm{~m} / \mathrm{s}$ and the heat exchanger tends to a new \\
& steady state $\left(b_{i j}\right.$ are as in Set 2$)$ \\
& flow arrangement Case 4, constant inlet temperatures, the initial \\
& state is the steady state, step temperature increase at the inlet to channel 3 \\
& by $5^{\circ} \mathrm{C}$
\end{tabular}

jump at the edge of the heat exchanger. Note that according to the formula (18), the predicted steady state time for Examples 1-3 is common which is

$$
\begin{aligned}
t_{s} & =\max \left\{\frac{2(1+0.6)}{1 \times 0.6}, \frac{2(0.5+0.6)}{(0.5 \times 0.6)}, \frac{2(0.5+1)}{(0.5 \times 1)}\right\} \\
& =\max \left\{\frac{2(0.7+0.6)}{0.7 \times 0.6}, \frac{2(0.5+0.6)}{(0.5 \times 0.6)}, \frac{2(0.5+0.7)}{(0.5 \times 0.7)}\right\} \\
& \approx 7.33,
\end{aligned}
$$

this is verified by the Figs. 2-4. 


\section{Conclusions}

The partial differential equations for the general case of the three-fluid parallelchannel (co- and counter-flow) heat exchanger have been solved analytically by formulating them into boundary control systems. Based on the analytical solution formula, the steady state and the time to reach the steady state are derived. The obtained steady state time formula is verified by the numerical simulations. However, we did not implement numerical calculations using the analytical solutions which are given in an abstract setting and we are not aware of a feasible algorithm. In addition, it is needed that the input function $u_{i}(t)$ is twice continuously differentiable which is strong in practical application. The relaxation of this regularity condition deserves further study.

Acknowledgement. The authors are indebted to the anonymous reviewers and the editors for helpful comments and suggestions. The second author was supported financially by the NSF of China grant 11501189, the Hunan Provincial NSF Project grant 12JJ4001 and the Scientific Research Foundation for the Returned Overseas Chinese Scholars, State Education Ministry.

\section{References}

1. Bielski S, Malinowski L (2005) An analytical method for determining transient temperature field in a parallel-flow three-fluid heat exchanger. International Communications in Heat and Mass Transfer 32: 1034-1044

2. Chen JH, Malinowski L (2014), Steady state of three-fluid parallel-flow heat exchanger system. Journal of Engineering Mathematics 87: 153-165

3. Chen JH, Lu WY (2011) Perturbation of nilpotent semigroups and application to heat exchanger equations. Appl Math Letters 24: 1698-1701

4. Curtain RF, Zwart H (1995) An Introduction to Infinite-Dimensional Linear Systems Theory. Springer-Verlag, New York

5. Grabowski P (2007) Stability of a heat exchanger feedback control system using the circle criterion. International Journal of Control 80 (9): 1388-1403

6. Hoffman JD, Numerical Methods for Engineers and Scientists, Second ed., Marcel Dekker, Inc., New York, 2001, pp. 437-447.

7. Kunimatsu N, Sano H (1998) Stability analysis of heat-exchanger equations with boundary feedbacks. IMA Journal of Mathematical Control \& Information 15: 317-330

8. Luo X, Guan X, Li M, Roetzel W (2003) Dynamic behaviour of onedimensional flow multistream heat exchangers and their networks. International Journal of Heat and Mass Transfer 46: 705-715

9. Malinowski L (2003) Equations for transient behaviour of parallel-flow multichannel heat exchangers. Heat and Mass Transfer 39: 321-325

10. Malinowski L, Bielski S (2009) Transient temperature field in a parallelflow three-fluid heat exchanger with the thermal capacitance of the walls and the longitudinal walls conduction. Appl Thermal Engineering 29: 877-883 
11. Mills AF (1992) Heat Transfer. IRWIN, Boston

12. Pazy A (1983) Semigroups of Linear Operators and Applications to Partial Differential Equations. AMS 44, Springer, New York

13. Sano H (2007) Observability and reachability for parallel-flow heat exchanger equations. IMA J. Math. Control and Inform. 24: 137-147

14. Sano H (2007) On reachability of parallel-flow heat exchanger equations with boundary inputs. Proc. Japan Acad. 83 (Ser. A): 1-4

15. Shrivastava D, Amel TA (2004) Three-fluid heat exchangers with three thermal communications. Part A: general mathematical model. International Journal of Heat and Mass Transfer 47: 3855-3867 

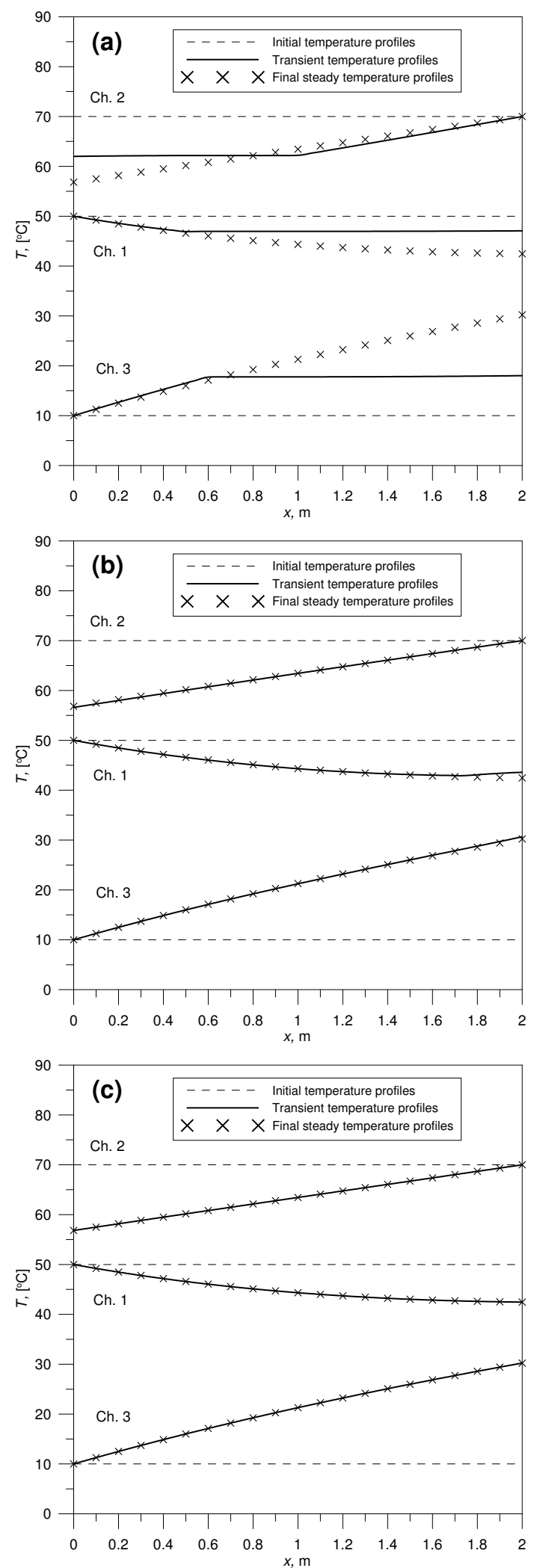

Fig. 2 Temperature profiles for Example 1. (a) $t=1 \mathrm{~s}$ (b) $t=3.5 \mathrm{~s}$ (c) $t=7.33 \mathrm{~s}$ 

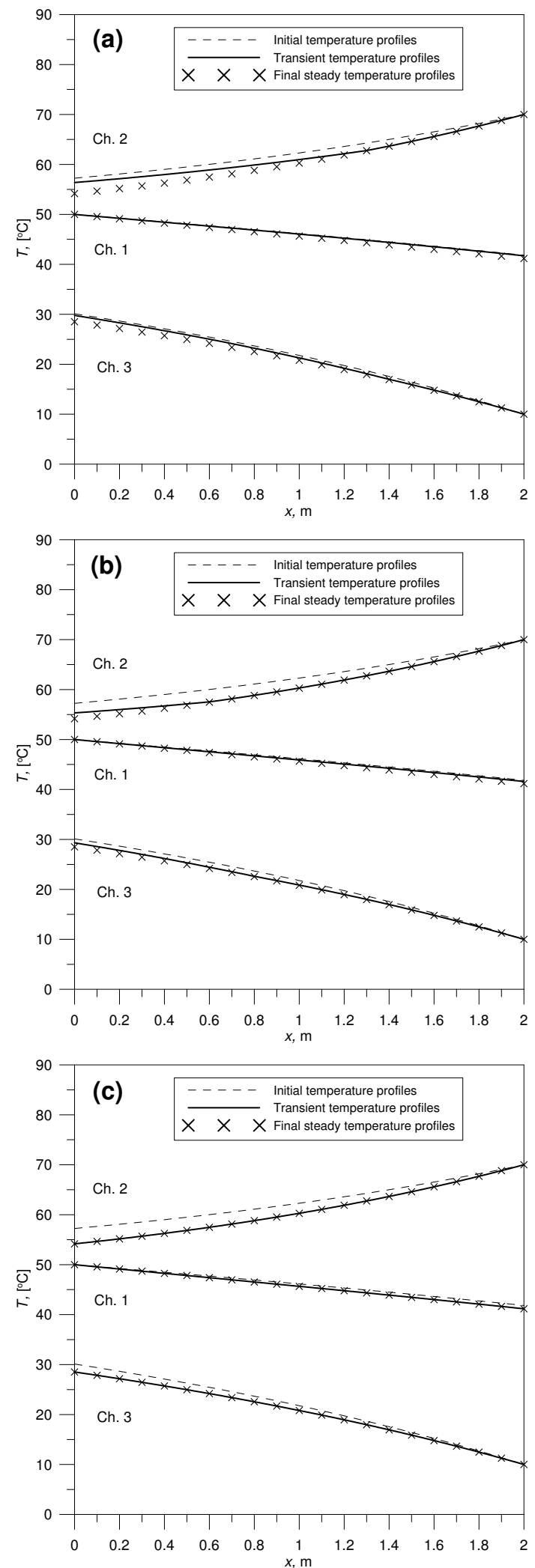

Fig. 3 Temperature profiles for Example 2. (a) $t=1 \mathrm{~s}$ (b) $t=2 \mathrm{~s}$ (c) $t=7.33 \mathrm{~s}$ 

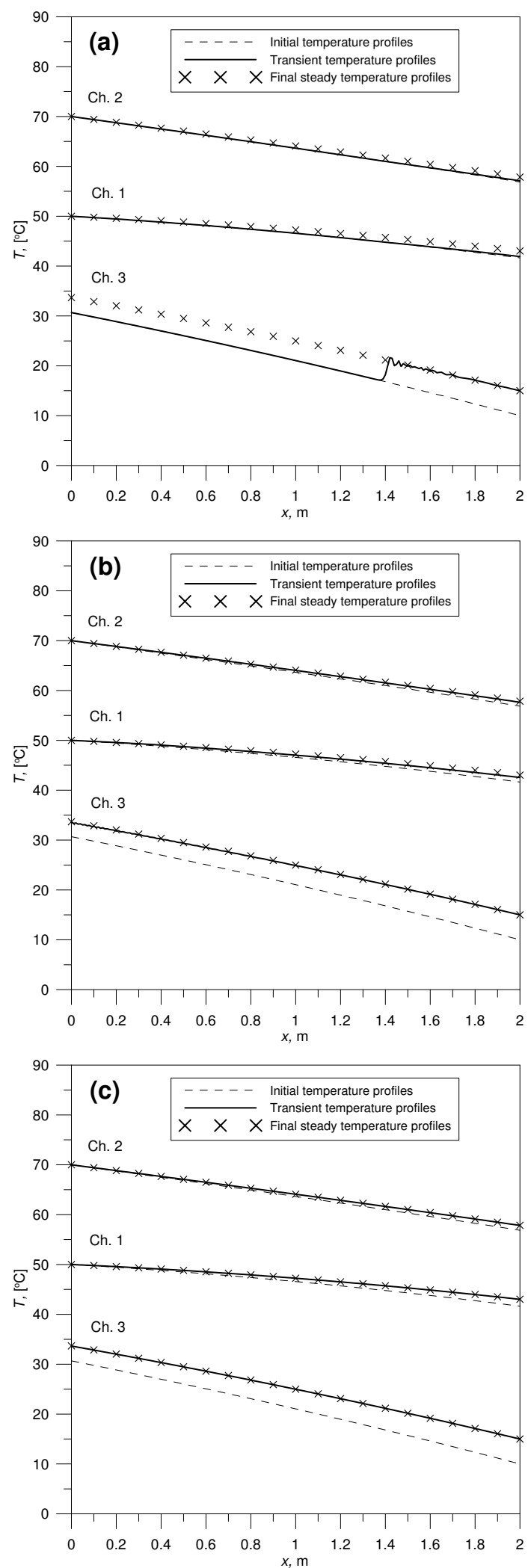

Fig. 4 Temperature profiles for Example 3. (a) $t=1 \mathrm{~s}$ (b) $t=4 \mathrm{~s} \quad$ (c) $t=7.33 \mathrm{~s}$ 